septic measures are rigorously attended to, reports show that the results are very satisfactory.

Tracheotomy.-This operation is performed to relieve asphyxiating symptoms caused by acute laryngitis or pharyngitis, by the presence of some foreign body in the pharynx or larynx, and in some cases if dangerous symptoms become manifested during the administration of some general anæsthetic.

The prognosis is usually good, particularly in cases where the tube is only to be worn for a short time.

The patient is placed in the dorsal position with the head well thrown back and the skin of the neck held as tight as possible; after the usual antiseptic precautions an incision is made in the upper third

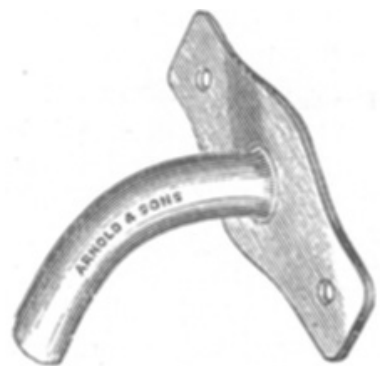

FIG. 51.

Tracheotomy tube.

of the neck about an inch below the larynx, at a spot where the trachea can be felt most superficially; the latter is picked up with a sharp curved hook or bent needle and thread, and an elliptical or circular piece excised to make a hole sufficiently large for the insertion of the tube. The tube is then placed in the trachea and fixed in position.

For temporary purposes a piece of bent glass tubing, held in position by tapes tied around the patient's neck, answers very well.

(To be continued.)

\title{
THE PATHOLOGY AND TREATMENT OF SPAVIN: A CRITICISM.
}

By James Macqueen, F.R.C.V.S., Royal Veterinary College, London.

THE September (I 898 ) issue of this Journal contains a translation of a masterly paper by Dr Eberlein, Berlin, on the pathology and treatment of spavin. The author devotes considerable space to a highly interesting abstract of the literature on the subject, but it may be noted that he quotes only two English authorities-Blaine (1805) and Smith (I 893)-although Gibson, Coleman, Goodwin, Percivall, Dick, and Williams have recorded opinions which deserve some recognition. For his time, I754, Gibson had a fairly satisfactory knowledge of spavin disease, and in respect of its treatment he anticipated much of current practice. Coleman stated that spavin originated in splint of 
the hind leg, an opinion which evoked the criticism that his pathology was both defective and erroneous. Goodwin, veterinary surgeon to George IV., gave the first demonstration in this country of occult spavin, and held that the disease generally commences between the two cuneiform bones, extending later into their substance, which becomes carious, and that exostosis is an after effect. Percivall states that the seat of spavin is on the cuneiform and scaphoid bones, in the joints which they form with each other and with the metatarsus below and astragalus above, that the inflammation originates in the ligamentary and periosteal tissues, and that once exostosis has formed there seems hardly any limit to its extension. Dick considers that in bone spavin the ligaments and cartilages have probably been injured, and in consequence the bone and periosteum "inflame and throw out ossific matter." Williams regards spavin as an inflammation of the cuneiform and metatarsal bones induced either by concussion or by extension of inflammation of the interosseous ligaments, which are always implicated in the morbid process, and, further, that the ostitis originates in the cancellated tissue, and that consecutive exudation perverts the nutrition of the articular cartilage, which ulcerates and disappears.

Eberlein, after investigating the condition of 120 diseased hocks, concludes that in spavin the first change, as a rule, is an osteoporosis (rarefying ostitis) of the scaphoid, cuneiform, or metatarsal bone, which is very quickly followed by an osteo-sclerosis. Then the disease attacks the articular cartilage, inducing chondritis, and sooner or later anchylosis, and frequently the inflammatory process extends from the joint or direct from the bone to the periosteum, and there sets up periostitis with the formation of exostosis. In other words, that spavin disease originates always in the bone substance, and gradually extends from below upwards or from within outwards, that exostosis is a later effect of the extending ostitis, and that articular disease is the result of antecedent change in the bone. This view of the origin and extension of spavin is supported by numerous drawings of diseased tarsal bones; and if one were content to accept without question Eberlein's interpretation of the lesions, further inquiry should be unnecessary. But anyone who will take the trouble to carefully examine $\dot{a}$ few spavined hocks and to compare these with a few healthy hocks, will soon discover the need for caution in assuming that the nature of sparin disease has been settled. It should be stated, however, that if Eberlein has rot succeeded in placing the pathology of spavin beyond dispute, he has earned high commendation by his zeal and industry in carrying out what must have been a laborious investigation.

Describing very minutely the macroscopical lesions revealed by vertical sections of spavined hocks, Eberlein finds spots or patches of an intense red colour (areas of ostitis), and the affected surface so soft as to yield to the pressure of the finger. These changes, he affirms, are observable in the earliest stages of the disease, "Iong before any alterations are visible in the articular cartilage or in the outer parts of the joint." This statement is pure speculation, unsupported by proof, and prima facie it is insupportable, for no one may predict from a red patch in a tarsal bone the occurrence of articular disease or spavin exostosis. Eberlein seems to attach 
unwarrantable importance to the colour of sections, which may be red or yellow and yet healthy. Much depends upon whether or not the horse has been bled at death. Softness of the coloured parts cannot be a constant feature of spavined hocks. Sections examined immediately after death do not yield anywhere to finger pressure, and even in the earliest stage of visible articular disease it is exceptional to find the substance of scaphoid, cuneiform, or metatarsal abnormally soft. The most frequent, but by no means constant, alteration of the interior of the affected bones is one of increased hardness and density, which may rightly be regarded as corresponding with the second stage of ostitis. Eberlein maintains that diagnosable spavin is always preceded by osteo-porosis. It may be so, though specimens showing this change are exceedingly difficult to find. But, granting provisionally that ostitis-rarefying and condensing-is the rule in spavin, how shall the exceptions be explained? Sections of typically spavined hocks are sometimes neither abnormally porous nor dense, and, except at the articular and free surfaces, remarkably like sections from healthy hocks.

Regarding the mode of extension of the disease from bone substance to articular surface or to the periphery, Eberlein states that the rarefying process extends indifferently in all directions, and involves the surrounding parts ; that it meets with resistance from the articular cartilage beneath which it spreads-interfering with nutrition and leading eventually to destruction of the cartilage. Undoubtedly when ostitis extends in the manner just indicated the cartilage will suffer, and so far the process of extension and destruction is quite feasible. But something more is required to explain the almost constant occurrence at one place of the earliest articular lesion. Specimens seem to prove that the resistance of the cartilage to destruction, however induced, is less towards the inner articular margin of scaphoid and cuneiform than elsewhere, and it may be asked why this should be so.

It is obvious that the destructive process in the cartilage must start somewhere, although an ostitis which extends indifferently in all directions hardly accounts for the almost invariable position of the earliest change. If the arthritic disease always resulted in complete disappearance of the cartilage, the situation or extent of the first breach of surface would have less importance, but the very limited articular lesion of many chronic cases furnishes a difficulty in spavin disease which no published theory of extension seems able to surmount. Another peculiarity not yet explained is the escape of the periosteum in occult spavin. Why the extending ostitis should not always invade the periosteum, which is in vascular communication with the area of inflammation, Eberlein does not venture to state, but he suggests that when the disease arises in the metatarsus it always extends upwards. Why not sometimes downwards? There is nothing in the structure of the large metatarsal bone to prevent distal extension of the rarefying process.

Ostitis is not the simplest disease, and shreds or microscopic sections of decalcified bone do not give unmistalsable information. Observation, perhaps, has been at fault. The boundaries of an inflamed area can generally be asccrtained, but the direction of extension within the area is not always ascertainable, especially in bone 
spavin. A probable explanation of most cases is, that the ostitis is superficial and reactionary, and that it constitutes an attempt to repair the effect of damage sustained at the articular or periosteal surface.

Objection may be taken to the statement that the earliest changes in the articular surface almost always occur $2-3 \mathrm{~mm}$. within the joint margin. In many cases the margin itself first shows disease, the remainder of the cartilage being free from spot. Bearing upon this point, Eberlein makes a discovery which unfortunately cannot be confirmed. He finds that the closing or contact edge of the smaller tarsal joints is from $2-3 \mathrm{~mm}$. within the articular margin, and to this "striking fact" he attributes the position of the first erosion of the cartilage. And further, that the contact here resembles that which obtains in the cardiac valves. But in hocks-healthy or diseased-the articular edges respond to one another with remarkable exactitude -a mere seam indicating the line of closure-which is certainly very unlike that of the cardiac valves.

Of the other changes in spavined hocks little need be said. They vary considerably. Eberlein describes punctiform projections (calcified points) as common features of the articular disease, but he does not mention cribriform erosion. Holes with smooth rounded margins usually occur and very early in the facets, and projections or spiculæ when present are generally confined to the insertion surfaces.

Anchylosis in many cases is limited, cartilage being retained on part of the facets. Fusion of scaphoid and cuneiform without a trace of the articular cleft occurs now and then, and most frequently in cases complicated by exostosis. Anchylosis is oftener peripheral than central, although at first view union may appear central owing to ossification of the interosseous ligaments. On breaking an anchylosed scapho-cuneiform joint, the articular surfaces may be found eroded yet free; the insertion surface of one side may show bony projections, and the other excavations extending into or threatening invasion of the adjacent facet. In a very ingenious description of the process of anchylosis, Eberlein states that the erosion of the cartilage develops at corresponding points of the opposed surfaces, but he does not very clearly explain how this happens. Proliferating granulations growing from the bone first eroded into the opposite surface may effect a correspondence of prominences and excavations, but the explanation hardly applies when both surfaces are similarly pitted or excavated, or when two bones, as scaphoid and cuneiform, simultaneously affected with ostitis exhibit articular lesions which resemble each other.

Regarding the nature of the joint affection, Eberlein rightly differs from those who hold that it is akin to the chronic deforming arthritis of the human subject, which attacks the larger and less frequently the smaller articulations. In man loose bodies occur within the affected joints, and the bones undergo deformation and shortening, but anchylosis has not been observed. In spavin arthritis the process, however induced, leads to anchylosis, and further investigation will probably prove that Schrader was right in defining spavin as "a peculiar independent joint disease."

The etiology of spavin is still obscure. Bruising of the smaller bones has been given as the initial change, and over-exertion of the hock in kicking, jumping, slipping, or in starting heavy loads as 
the chief external cause. Experience teaches that spavin disease or lameness frequently follows wrenching or other similar injury during hock extension, but it has not been determined that accident of the kind indicated inflicts more damage to bone tissue than to the other structures of the hock. In the action of joints the bones cannot be considered independently of the cartilage, and it is not improbable that both cartilage and bone are injured at the same time. In the compression of the tarsal bones which is believed to result in spavin the first effect of the pressure should be felt by the cartilage, which will not withstand repeated squeezing unchanged. It has been ascertained that simple incised wounds of cartilage can be repaired, and that bruising leads to erosion or to transformation of the cartilage into bone. The progress of the arthritic disease is remarkably slow, and in many cases the horse is not lame and spavin may not have been suspected.

The causa interna is still more difficult to understand, but the custom has been to ascribe cases of spavin to inherent weakness of the hock. Eberlein pretends to recognise in the complicated construction of normally built hocks a special predisposition to mechanical injury, though he does not indicate what he means by a normally built hock. All hocks may be said to be complicated in construction. Hoclis of every conformation may be found spavined, and, although small or weak, flat or thin tocks are more often affected, satisfactory explanation of the cause of spavin disease must be sought in something else than mere shape. It is true that horses with long bodies, heavy quarters, and light legs, whether cow-hocked, straight or bandy legged, are sometimes affected, but horses with short bodies, light quarters, and strong legs, and even cattle-old cows in particular -are frequently spavined. If horses of high courage and free action often suffer, horses that are sluggish or exceptionally quiet and without attractive action do not escape. Observation suggests that the class of work has an important share in the causation of hock lameness, and that 'bus and other horses, doing work that necessitates incessant stopping and starting on roadways which give only an indifferent foothold, furnish a large proportion of cases of spavin.

The almost invariable occurrence of the disease at the inner side of the hock has been variously explained. One view is that the inner parts carry more of the weight than the outer; and another that the screw-like form of the articular ridges of the astragalus serves to conduct the pressure during hock extension more to the inner side. The value of these observations Eberlein, forgetting Goodsir, credits to Hering and Prosch. But careful examination of the tarsal bones, with regard to position, prominence, and curvature of the articular facets, should assist in arriving at the probable cause of the incidence of spavin at the inner part of the joint and of the situation of the usual articular lesion. In normal scaphoid and cuneiform bones the cancellated tissue exists chiefly within the ligament insertion surface, where compact bone is thinnest and contact is less intimate than at the facets. The earliest visible articular lesion usually appears on the antero-internal part, where compact bone is thickest, and, presumably, where resistance is strongest. If Eberlein is right the causal bruising affects the strongest part, and the resulting rarefying ostitis extends through dense bone and not in the direction of least 
resistance. If ostitis always initiates the disease, the insertion surfaces should more frequently show its effects. But evidently the ostitis, like the arthritis, is peculiar, and it is not easy to demonstrate the different stages of either process. A reasonable solution of the difficulty may be found by supposing that both arthritis and ostitis begin simultaneously, and that sometimes the articular disease is more active and extensive than the ostitis.

In explaining the extension of chronic joint disease it is always safe to assume the existence at one time or another of rarefying or condensing ostitis, which, as a superficial process, is a constant accompaniment of chronic ossifying periostitis. But it must be said that neither the excentric nor the concentric theory of extension will satisfactorily account for all cases of spavin. Specimens prove that there is more than one way, and, indeed, it would not be difficult to find hocks to illustrate and more or less prove four of the five recognised theories of the origin and extension of sparin disease.

To meet the latest theory of the genesis of spavin it will be necessary to revise the symptomatology, and to consider the possible existence of, (I) spavin disease without either lameness or exostosis, (2) spavin with exostosis but without lameness, (3) spavin without exostosis but accompanied by lameness, and (4) spavin with both lameness and exostosis. The first variety may be distinguished as Eberlein's spavin, in which ostitis of one or another of the tarsal bones occurs long before visible alteration of the articular cartilage or of the outer parts of the joint. No symptoms are exhibited in the living animal. This sort of spavin is distinctly occult, and altogether outside ordinary practice. The second is common enough, and the third constitutes a variety of tarsal disease which, in most cases, is more difficult to discover than any other. The fourth usually presents no special difficulty in diagnosis.

The symptoms described by Eberlein include all those usually accepted and one or two that have little value. The spavin test is not reliable even in cases where examination of the limb in other ways yields negative information. To distinguish a coarse hock from spavin exostosis is especially difficult, but veterinary surgeons who take a lenient view of enlarged hocks-which are only suspicious because of coarseness-seldom have occasion to regret their opinion. Diagnosis by measuring suspected hocks is unlikely to meet with approbation outside veterinary schools, and the employment of the Rontgen rays as an aid to diagnosis, or to lessen difficulties which too often are much exaggerated, will have to wait further improvement in the application of the apparatus. With Eberlein the prognosis of spavin is always uncertain and the presence of exostosis gives a permanent predisposition to lameness. This is an extreme view but it is undoubtedly safe, for the reason that no one can say how long a horse, bought sound in action, will continue at work free of lameness. Obcervation, however, goes to show that horses with spavined hocks may work for years without falling lame; indeed one of the several enigmas of spavin is the continuance of unexceptionable action. But when lameness exists only a rash mind will undertake to fix the date of its disappearance. Statistics of the curability of spavin lameness are of trifling value; their accuracy is only problematical, as it must be so long as diagnosis is uncertain. 
Eberlein seems to think that the lameness engenders other diseases in the affected leg, and cites ringbone, inflammation and contraction of the flexor tendons, and disease of the stifle joint, as sometimes following'spavin. In attributing these diseases to the existence of spavin lameness he goes beyond probability or draws from a unique experience. If there is any truth in the compression theory of the causation of spavin, ringbone and strained flexor tendons are more likely to precede than follow spavin disease, and this can be affirmed of ringbone.

The natural tendency in spavin is towards consolidation of the affected bones, and the value of treatment mainly depends on whether it promotes or retards anchylosis. For nearly a century and a half the treatment of spavin has undergone little change except in the method of application, which has been modified and perhaps improved. Line and puncture firing, caustics, setons, tenotomy, periosteotomy, slitting the cunean bursa, and even neurectomy-the last resort of the baffled surgeon-have all been practised. With one exception the least reasonable of these operations is cunean tenotomy, which seldom confers benefit, because in the majority of cases the tendon passes above the enlargement. Periosteotomy, first practised by Sewell ( 1835 ), is a bolder operation, and one that is indicated when exostosis is very large. Setons are still useful, and, if not quite so beneficial as periosteotomy, they are far less risky. Piercing the exostosis with a sharp fleam-headed iron was practised by Gibson, Coleman, and Turner before the introduction of the iron with conical head or needle-shaped point. Eberlein strongly recommends perforating firing. He fired IO2 horses and in many effected a curewhich must be extremely gratifying to those who puncture exostoses. It is to be regretted that Eberlein is silent as to the treatment of lameness due to occult spavin, of which he must have large experience. At one time occult cases were treated by repeatedly blistering the hock and resting the horse in loose-box or straw yard for six months; but in this neurotic age owners too often lack patience and refuse to submit their lame horses to treatment that is only expectant. On humane grounds succedaneous rest needs no apology, but it cannot be defended as promoting anchylosis, which should be the aim of the treatment of spavin.

\section{EXPERIMENTAL TUBERCULOSIS IN THE ASS AND THE EFFECT OF TUBERGULIN.}

\section{By Stewart Stockman, Professor of Pathology, Royal Dick Veterinary College, Edinburgh.}

For a time the existence of tuberculosis in the horse tribe was denied, and the denial was based both on clinical and experimental observations. Later clinical experience, however, has shown that the disease in the horse is by no means rare in this country at least, and the more recent experiments prove that the horse can be infected artificially with tuberculosis, although positive results seem to depend on certain provisions. Some of these conditions are evident, others call for further investigation. The experiments which I am about to describe seem 